\title{
PERIPHERAL COMPONENT INTERCONNECT EXPRESS CARD FOR THE CLOSED-CIRCUIT TELEVISION APPLICATION
}

\author{
Vaclav Mach, Milan Adamek Jan Valouch, Ondrej Zimek \& Marta Blahova
}
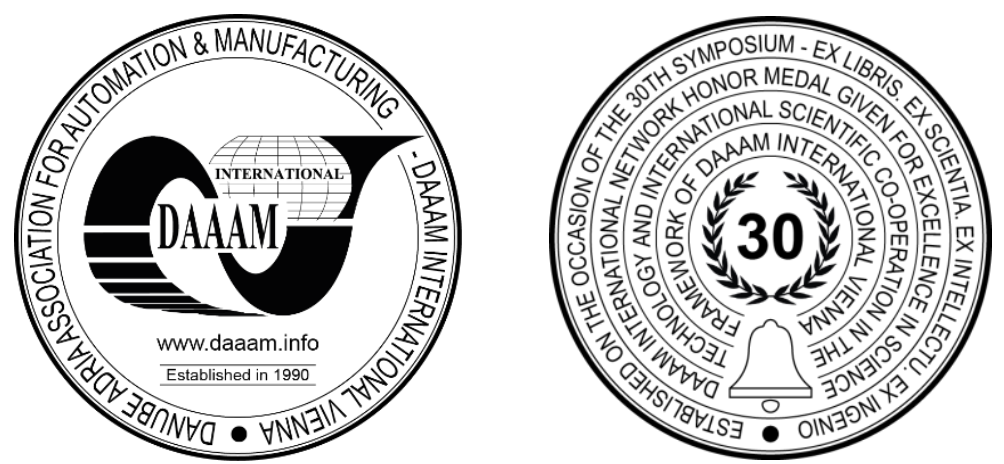

This Publication has to be referred as: Mach, V[aclav]; Adamek, M[ilan]; Valouch, J[an]; Zimek, O[ndrej] \& Blahova, M[arta] (2019). Peripheral Component Interconnect Express Card for the Closed-Circuit Television Application, Proceedings of the 30th DAAAM International Symposium, pp.0605-0609, B. Katalinic (Ed.), Published by DAAAM International, ISBN 978-3-902734-22-8, ISSN 1726-9679, Vienna, Austria

DOI: $10.2507 / 30$ th.daaam.proceedings.082

\begin{abstract}
Technical security became very popular and convenient in many sectors where physical security is not a sufficient solution. Many companies use the Closed-Circuit Television (CCTV) application for security applications. The aim of this paper is to design the extended Peripheral Component Interconnect Express (PCI-E) card for the CCTV application for extensive companies. The card has three independent Ethernet terminal where IP cameras can be connected. This solution is much more cost-effective and easy to use solution than traditional Digital Video Recorder (DVR). The card communicated via the Universal Serial Bus (USB) interface which is implemented in the PCI-E standard. Designed solutions guarantee flawless and reliable video signal transmission for the security applications.
\end{abstract}

Keywords: Technical Security; Closed-Circuit Television; Ethernet; Peripheral Component Interconnect Express.

\section{Introduction}

The Intruder Alarm System (IAS) became very popular in modern households where people want to protect their possession effectively. This system can be classified as technical security which replaces the less efficient physical security. The physical security is very often used in large buildings or areas where the person can control several systems; However, this manner is very inefficient and expensive in small family houses. The author [1] mentioned that a person stares at a screen for more than 20 minutes, his attention drops by 30\%; and for periods over an hour, this drop can reach $70 \%$. That's why the IAS is much more suitable and also more cost-effective for the small family houses. The standard IAS consists of several devices which can detect the intruder in the protected area without any person involved.

Every IAS is directly connected to the regional Alarm Receiving Centre (ARC) which can react to the triggered alarm message. [2] When the alarm is triggered, the employs must be able to distinguish between the real alarm and the false alarm. This decision must be very quick and infallible. Nowadays, the ARC and the IAS can use the live camera view to the protected area which can be very helpful for the employers of the ARC. [3] The residential areas have typically one or two cameras in the building which is sufficient. However, bigger buildings or companies can use up to hundreds of security cameras using the Ethernet connection. 
All cameras are usually connected to the server which is not always the best decision. According to the author [4], the best way how to connect security cameras for live monitoring by the person is the direct connection. The most usual monitoring device is still the Personal Computer (PC) which offers a lot of advantages and also a very friendly interface for the management. [5] The Ethernet connection in standard PC has the bottleneck which is the capacity of the connection. There are some situations, that one Ethernet port is not enough and the live video from connected cameras starts to jamming. This problem can be solved by using more Ethernet ports on the motherboard or by adding several extension Ethernet cards. According to this problem, the main goal of this article is to design the special extension card with Ethernet ports which can be connected to the motherboard using the PCI-E slot.

\subsection{Peripheral Component Interconnect Express}

The Peripheral Component Interconnect Express (PCI-E) is a high-speed serial computer expansion bus standard, which has characteristics of high speed, low power, and high protocol efficiency. [6] This standard is widely used as a standard I/O interface for connecting processors and I/O system devices. High speed, low power, and high efficiency are the salient properties of the PCI-E; because of additional properties, PCI-E is considered as good alternatives to the existing network structures. [7]

It also uses a bus topology to enable communication between other devices on the bus, and it supports multiple lanes of 1x, 2x, 4x, 8x, 16x, and 32x per link. Data rates are $2 \mathrm{~Gb} / \mathrm{s}$ per lane in PCI-E Gen 1, 4 Gb/s per lane in PCI-E Gen 2 and $8 \mathrm{~Gb} / \mathrm{s}$ in Gen3, and the bandwidth is $128 \mathrm{~Gb} / \mathrm{s}$ and the clock speed is $8 \mathrm{GHz}$ based on the PCI-E Gen3 16x lane. [8] Every PCI-E slot has a switch which is a collection of logically connected PCI-PCI bridges. After connecting the additional PCI-PCI bridges downstream, one PCI-PCI bridge is upstream. It means that switching appears as a hierarchical structure of logical PCI bridges [8]. PCI-E is one example of the general trend toward replacing parallel buses with serial interconnects like Universal Serial Bus (USB). PCI Express is a serial connection that operates more like a network than a bus. Instead of one bus that handles data from multiple sources, PCI-E has a switch that controls several point-to-point serial connections.

\section{Physical Design of the Extension Card}

The physical design of the card must follow the standardization for the dimensions and power management for the PCI-E cards. On the board must be placed all needed components for the reliable operation. On the side which is facing out of the PC case are placed Ethernet terminals with the proper signalization. On the internal side are placed also the terminal for the power which is distributed by the PC power source.

\subsection{The Ethernet chip LAN7500i}

Used Ethernet chip is called LAN7500i which consists of a high-performance Hi-Speed USB 2.0 to 10/100/1000 Ethernet controller. With applications ranging from embedded systems, set-top boxes, and PVR's, to USB port replicators, USB to Ethernet dongles, and test instrumentation, the device is a high performance and cost-competitive USB to Ethernet connectivity solution. The LAN7500/LAN7500i contains an integrated 10/100/1000 Ethernet MAC and PHY, Filtering Engine, USB PHY, Hi-Speed USB 2.0 device controller, TAP controller, EEPROM controller, and a FIFO controller with a total of $32 \mathrm{~KB}$ of internal packet buffering. The device implements Control, Interrupt, Bulk-in, and Bulk-out USB Endpoints. The Ethernet controller supports auto-negotiation, auto-polarity correction, HP Auto-MDIX, and is compliant with the IEEE 802.3, IEEE 802.3u, IEEE 802.3ab standards. An internal EEPROM controller exists to load various USB configuration information and the device MAC address. The integrated IEEE 1149.1 compliant TAP controller provides a boundary scan via JTAG.

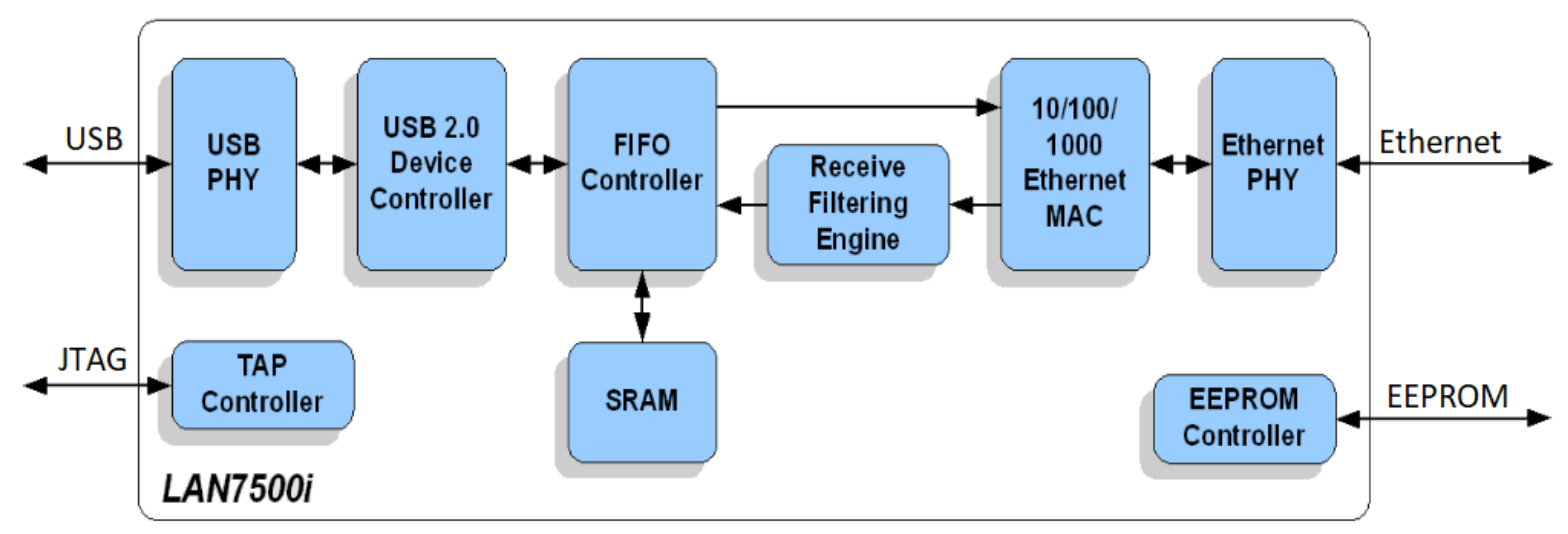

Fig. 1. Block schematic of the Ethernet chip LAN7500i. 
The chip can be programmed by the TAP controller which means that using this connection the chip can be programmed by the user during the operation. This interface can be connected to the other controller which can communicate via the USB interface and the user can program the chip without any disconnecting the card.

\subsection{Schematic design of the card}

The main chip of the board is the LAN7500i which must be powered by several voltages. Due to this problem, several voltage regulators are placed near each chip. This voltage must be smoothed by the capacitors and filtered by the chokes. The critical part of the connection Is the physical Ethernet connection. The physical Ethernet connection needs Ethernet Magnetic Transformers which can transform the signals from the LAN7500i to the physical link of the Ethernet interface. These transformers offer high isolation for robust applications, it provides safety isolation of up to $6 \mathrm{kV}$ which is ideal for technical applications.

The second problem comes with the connection to the PC. On the board must be a communication interface that can communicate with all Ethernet chips via the PCI-E. The standard interface for the communication is done via the USB which is used in this scenario. The PCI-E slot has a complete USB interface using all USB 3.0 features. The pins HSOp(0) and HSOn $(0)$ are responsible for the transmitting part of the channel and the pins HSIp (0) and HSIn (0) are responsible for the receiving part. There are also pins for the USB 2.0 REFCLK+ and REFCLK-. All mentioned pins can be used for further communication via the PCI-E. The main part of the schematics for this article can be seen in the following figure.

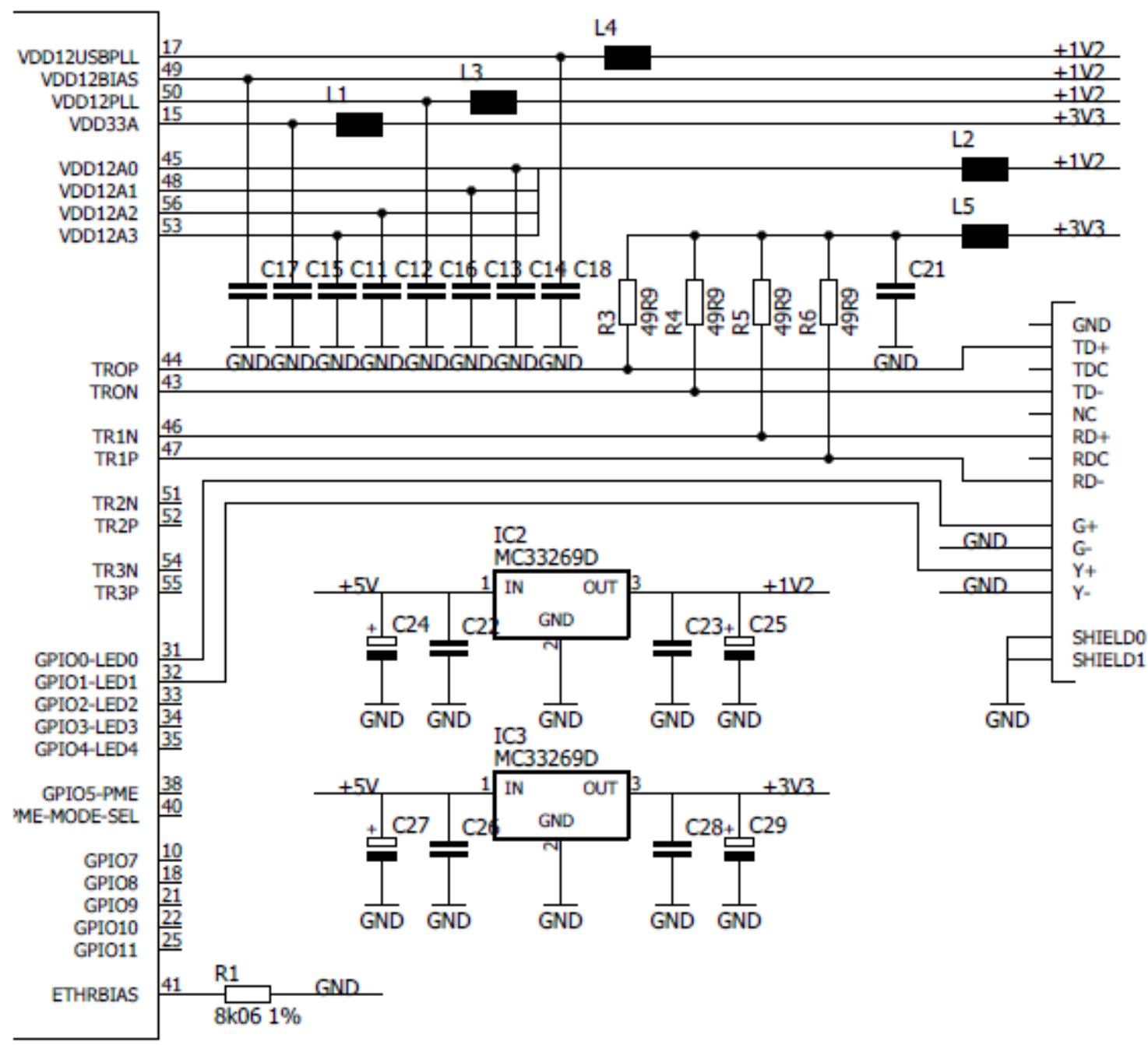

Fig. 2. The main schematic part with the physical Ethernet connection.

For the USB hub which is able to communicate via the PCI-E interface was chosen the model USB5534B. The USB5534B hub is a 4-port SuperSpeed/Hi-Speed, low-power, configurable hub controller family fully compliant with the USB 3.0 Specification. All required resistors on the USB ports are integrated into the hub. This includes all series termination resistors and all required pull-down and pull-up resistors on D+ and D- pins. The over-current sense inputs 
for the downstream facing ports have internal pull-up resistors. That meant that the USB hub does not need any external components for the flawless and reliable operation.

\section{The final design of the card}

The final design of the PCI-E card has dimensions as a normal card and it is suitable for the standard PC case. The standard 1x PCI-E terminal is used. The card also has the external power source which must be connected by the standard Molex connector on the side of the card. Each Ethernet connector with the transformers has its own chip which can be independently programmed and each chip has own power management. There is also the USB hub USB5534B which spreads the one single bus to three, each for one Ethernet chip.

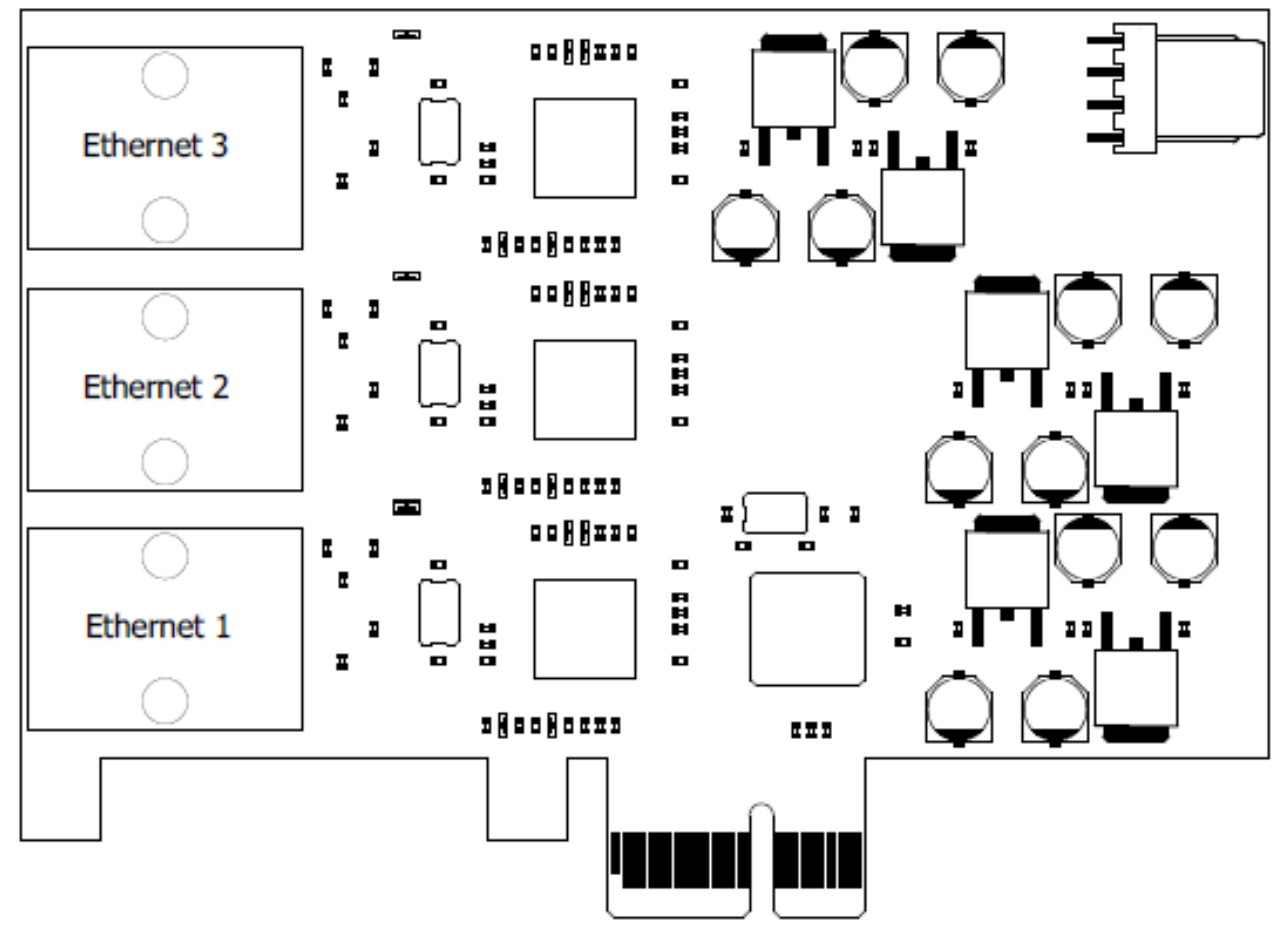

Fig. 3. The final design of the PCI-E card for IP cameras.

\section{Conclusion}

This paper presented a possible design of the extended PCI-E card for CCTV application which can be connected to the standard computer instead of DVR. The designed card is constructed according to standard for the PCI-E cards. Final PCB consists of three independent Ethernet terminal whit separate chips and power management. The final product can be used for large surveillance centers using hundreds of IP cameras. Several terminals guarantee a stable and reliable connection without any additional device. The extensional card needs the external power voltage for the proper operation which can be distributed by the PC power supply via the standard Molex connector. The chip for Ethernet communication does not need any additional program interface or special installation procedure and the system is plug-and-play.

The USB hub can be removed from the design in case of using at least PCI-E 4x where three independent USB lines can be found. Moreover, the PCI-E 4x can also help with the stability inside of the case. However, the used PCI-E 1x connector can be placed in any PCI-E connectors despite the numbers of lines, which makes PCI-E 1x the most universal. The further research can be also focused on the implementation of the Power over Ethernet (PoE) which can distribute the power for the cameras via the Ethernet cable. The next generation should have local data storage and also the realtime microcontroller for programming.

\section{Acknowledgments}

This work was supported by the Ministry of Education, Youth and Sports of the Czech Republic within the National Sustainability Program Project No. LO1303 (MSMT-7778/2014) and also by the European Regional Development Fund under the project CEBIA-Tech ED2.1.00/03.0089 and by the Internal Grant Agency of Tomas Bata University under the project No. IGA/CebiaTech/2019/00 and the IGA/FAI/2019/003. 


\section{References}

[1] Landa, J., Jun, Ch. \& Jun, M. (2017) Implementation of a Remote Real-Time Surveillance Security System for Intruder Detection. 9th International Conference on Measuring Technology and Mechatronics Automation (ICMTMA), doi:10.1109/icmtma.2017.0032.

[2] Valouch, J. (2015) The Proposal of Methodology for Evaluating the Effectiveness of Alarm Systems. Applied Mechanics and Materials. pp. 183-88. doi:10.4028/www.scientific.net/amm.

[3] Ševčík, J., Malus M. \& Svoboda P. (2014) Large-scale industrial company alarm receiving centre modernization design. WSEAS Transactions on Communications. pp. 587-595. ISSN 1109-2742.

[4] Li, W. \& Gao Y. (2018) Design of Network Camera Based on ONVIF in the Dark Environment. In: 2018 International Conference on Sensor Networks and Signal Processing (SNSP). pp. 84-87. DOI: 10.1109/SNSP.2018.00025. ISBN 978-1-5386-7413-0.

[5] Gao, Y., Ma, H., Liu W. \& Yu, S. (2016) Cost Optimal Resource Provisioning for Live Video Forwarding Across Video Data Centers. Springer International Publishing, pp. 27-38. DOI: 10.1007/978-3-319-42553-5_3. ISBN 9783-319-42552-8.

[6] Gabrielli, A., F. Alfonsi, G., Balbi, G., Damen, D., Falchieri, N., Giangiacomi \& R. Travaglini. (2019) A MultiChannel Pci Express Readout Board for Fast Readout of Large Pixel Detectors. Nuclear Instruments and Methods in Physics Research Section A: pp. 279-281. Doi: 10.1016/J.Nima.2018.06.080. ISSN 01689002.

[7] Shim, Ch., Shinde, R. \& Choi M. (2019) Compatibility Enhancement and Performance Measurement for Socket Interface with Pcie Interconnections. Human-Centric Computing and Information Sciences. Doi: 10.1186/S13673019-0170-0. ISSN 2192-1962.

[8] Ravindran, M. (2007) Cabled Pci Express-A Standard High-Speed Insterument Interconnect. IEEE Autotestcon. pp. 410-417. Doi: 10.1109/Autest.2007.4374248. Isbn 978-1-4244-1238-9. Issn 1088-7725. 